\title{
Aspectos éticos e normativos de um estudo clínico multicêntrico de oncologia pediátrica
}

Maryelle M L Gamboa ${ }^{1}$, Lauro J Gregianin ${ }^{2}$

\section{Resumo}

O artigo objetiva avaliar o cumprimento dos aspectos éticos e normativos de um protocolo de experimentação clínica multicêntrico em oncologia pediátrica. A análise do trâmite regulatório, bem como do processo de obtenção do termo de consentimento de 180 pacientes de 16 instituições, foi empreendida por meio das fichas clínicas dos pacientes. Dez dos dezesseis centros submeteram o protocolo ao comitê de ética em pesquisa local. Em relação ao termo de consentimento livre e esclarecido, 161 dos 180 pacientes consentiram e assinaram o termo aplicado pelo pesquisador. A coordenação do estudo compreende que houve algumas limitações relacionadas com estes aspectos, pois o estudo envolveu significativo número de instituições. Por este motivo, especialmente em projetos multicêntricos, uma monitoria mais rigorosa, tanto em termos de orientação e fiscalização do trâmite regulatório como no processo de obtenção do Termo de Consentimento Livre Esclarecido, poderia prevenir situações como as encontradas.

Palavras-chave: Sarcoma de Ewing. Consentimento livre e esclarecido. Ética. Serviço hospitalar de oncologia. Estudo multicêntrico.

\section{Resumen}

\section{Aspectos éticos y normativos de un ensayo clínico multicéntrico de oncología pediátrica}

El artículo tiene como objetivo evaluar el cumplimiento de los aspectos normativos y éticos de un protocolo de ensayo clínico multicéntrico en oncología pediátrica. El análisis del proceso regulatorio, así como el proceso de obtener el consentimiento de 180 pacientes procedentes de 16 instituciones se llevó a cabo a través de las historias clínicas de los pacientes. Diez de los dieciséis centros presentado el Protocolo para la Ética en la búsqueda local. En cuanto a caducidad de Consentimiento, 161 de 180 pacientes consentido y firmado por el investigador. Coordinación del Estudio entiende que hay algunas limitaciones relacionadas con estos aspectos, que ya se esperaba debido a que el estudio incluyó un número significativo de instituciones. Por esta razón, especialmente en proyectos multicéntricos, un control más riguroso en términos de orientación y supervisión del procedimiento regulador como en el proceso de obtención de la IC, podría evitar situaciones como las que se encuentran.

Palabras-clave: Sarcoma de Ewing. Consentimiento informado. Ética. Servicio de oncología en hospital. Estudio multicéntrico.

\section{Abstract \\ Ethical and normative aspects of a multicenter clinical study of pediatric oncology}

The article aims to assess compliance with normative and ethical aspects of a multicenter clinical trial protocol in pediatric oncology. The analysis of the regulatory proceeding, as well as the process of obtaining the Consent of 180 patients from 16 institutions was undertaken through the medical records of patients. Ten of the sixteen centers submitted the Protocol to the Institutional Review Board. Regarding Informed Consent, 161 of 180 patients consented and signed by the researcher. The Coordination Study understands that there are some limitations related to these aspects, which was already expected because the study involved a significant number of institutions. For this reason, especially in multicenter projects, a more rigorous monitoring in terms of guidance and supervision of the regulatory proceeding as in the process of obtaining the Informed Consent, could prevent situations such as those encountered.

Key words: Ewing, Sarcoma. Informed consent. Ethics. Oncology service, hospital. Multicenter study.

1. Mestre maryellelg@hotmail.com. 2. Doutor Igregianin@hcpa.ufrgs.br - Universidade Federal do Rio Grande do Sul (UFRS), Porto Alegre/RS, Brasil.

\section{Correspondência}

Maryelle Gamboa - Serviço de Oncologia Pediátrica, Hospital de Clínicas de Porto Alegre, Rua Ramiro Barcelos, 2350, $3^{\circ}$ andar leste CEP 90035-903. Porto Alegre/RS, Brasil.

Declaram não haver conflito de interesse 
Recentes dados epidemiológicos mostram que a sobrevida dos pacientes portadores de câncer infantojuvenil tem melhorado significativamente nos últimos 20 anos ${ }^{1,2}$. Entre os aspectos que mais contribuíram para a melhoria no prognóstico desta população estão o aumento no número de centros especializados equipados com unidades de terapia intensiva e de radioterapia; a disponibilidade de exames de imagem e patológicos mais acurados, bem como de antibióticos mais eficazes; o desenvolvimento de novas técnicas cirúrgicas e, principalmente, a utilização de tratamentos quimioterápicos mais eficazes e menos tóxicos identificados em estudos clínicos ${ }^{1}$.

Considerando que a incidência do câncer infantojuvenil é rara, dificilmente uma instituição atenderá isoladamente uma quantidade suficiente de pacientes para conduzir um estudo clínico randomizado e obter resultados cientificamente válidos em período razoavelmente curto de observação. Portanto, a atuação dos grupos cooperativos em oncologia pediátrica estimulando a inclusão de pacientes de diferentes instituições nestes estudos, sempre respeitando os conceitos que delineiam a pesquisa clínica, resultou na identificação dos tratamentos atualmente disponíveis.

Os primeiros estudos clínicos cooperativos internacionais na área da oncopediatria surgiram na década de 70, quando pesquisadores de vários centros de pesquisa recrutaram pacientes com a finalidade de aplicar e comparar diferentes esquemas de tratamento. Os resultados favoráveis observados nestes estudos foram incorporados à assistência, determinando melhora significativa nos índices de cura desta população. Entretanto, apesar dos altos índices de sobrevida entre os pacientes acometidos, os grupos cooperativos persistem na busca de esquemas terapêuticos mais eficazes, sempre por meio de estudos clínicos ${ }^{3,4}$.

No Brasil, a Sociedade Brasileira de Oncologia Pediátrica reconhece a necessidade de estudos cooperativos que considerem a nossa realidade. Por conseguinte, tem organizado e incentivado a formação de grupos cooperativos para os diversos tipos de tumores. A criação desses grupos visa a implantação de protocolos de tratamento e de pesquisa prospectivos, sempre considerando a realidade de cada centro, utilizando os recursos existentes nas diferentes instituições que atendem crianças com câncer em todo o território nacional.

Os estudos cooperativos são considerados de grande importância para o meio científico e caracterizam-se por serem simultaneamente executados em diversas instituições de pesquisa, conduzidas por diferentes pesquisadores e periodicamente supervisionados por monitores de pesquisa clínica. Durante as visitas de monitoria, esses profissionais são responsáveis pela verificação das informações geradas em cada centro de pesquisa e confirmação dos dados obtidos. Além disso, avaliam se o estudo clínico está sendo conduzido de forma adequada às Boas práticas clínicas ${ }^{5}$ e legislações aplicáveis.

\section{Normativas éticas de pesquisa}

Os aspectos éticos e legais relativos à participação de pacientes em estudos clínicos são assegurados, primeiro, pela aprovação da pesquisa clínica por comitê de ética em pesquisa (CEP); segundo, pelo processo de obtenção do termo de consentimento livre e esclarecido (TCLE), que detalha a natureza do estudo e descreve os potenciais benefícios, riscos e prejuízos ao paciente - que podem ocorrer durante a sua condução ${ }^{6,7}$.

A partir da Declaração de Helsinque ${ }^{8}$ estabeleceu-se a criação e implantação de CEP. No Brasil, a primeira resolução do Conselho Nacional de Saúde (CNS), a Resolução $1 / 88$, definiu a necessidade da existência dos CEP ${ }^{9}$. Posteriormente, a Resolução CNS 196/96 ${ }^{10}$ aprofundou os aspectos envolvendo a ética em pesquisa e estabeleceu que além dos CEP havia também a necessidade de criar a Comissão Nacional de Ética em Pesquisa (Conep), estabelecendo um sistema integrado de avaliação de protocolos de pesquisa. As normas de pesquisa em saúde, brasileiras ou internacionais, preveem que todos os projetos que envolvam seres humanos devem ser submetidos à análise e aprovação de um CEP antes de sua execução. A diretriz VII.14.a da Resolução CNS 196/96 descreve que a revisão ética de toda e qualquer proposta de pesquisa envolvendo seres humanos não poderá ser dissociada da sua análise científica ${ }^{10}$. Em 1995, levantamento realizado por Francisconi et al, referente aos CEP nacionais, verificou que entre os 26 hospitais brasileiros avaliados apenas 15 possuíam CEP local. Os autores lamentam a forma inadequada como a pesquisa clínica estava sendo realizada em algumas instituições e salientam a importância do conhecimento das legislações nacionais e internacionais e das atribuições dos CEP pelos investigadores ${ }^{11}$. No final de 2005, dez anos após o levantamento realizado por Francisconi et al, a Conep registrou 448 CEP no país, entre 650 solicitações ${ }^{12}$.

As normas éticas exigem que o paciente participante forneça seu consentimento livre e esclarecido, abordagem esta fundamental para preservar o direi- 
to de autonomia e poder de decisão em relação a sua participação no estudo clínico ${ }^{10}$.

Na definição estabelecida pelas Boas práticas clínicas ${ }^{5}$, o consentimento é um processo pelo qual um sujeito de pesquisa confirma a sua disposição em participar de um estudo clínico particular depois de ter sido informado de todos os aspectos da pesquisa que são relevantes para sua decisão. Este consentimento é documentado por escrito por termo de consentimento livre e esclarecido que deve ser preenchido e assinado ${ }^{13}$. Além disso, a Resolução CNS 196/96, na introdução da diretriz IV, diz que o respeito devido à dignidade humana exige que toda pesquisa se processe com consentimento livre e esclarecido dos participantes, indivíduos ou grupos que, por si e/ou por seus representantes legais, manifestem a sua anuência à participação na pesquisa ${ }^{10}$.

As informações referentes ao consentimento devem ser fornecidas de maneira verbal e por escrito, em linguagem compreensível. Adicionalmente, os pesquisadores responsáveis devem estar disponíveis para esclarecer eventuais dúvidas. O paciente convidado a participar do estudo pode necessitar tempo para conversar com seus familiares antes de decidir-se. Nesses casos, o pesquisador deve respeitar a solicitação do paciente. A assinatura do TCLE pelo paciente é a última etapa do processo e define o momento em que o participante documenta a sua autorização para participação no estudo clínico.

Ao longo dos últimos anos têm sido publicados diversos estudos nacionais e internacionais referentes ao vocabulário empregado e à estrutura do texto do TCLE utilizado nas pesquisas. Muitos destes trabalhos indicaram que o texto dos TCLE não é suficientemente entendido pelos pacientes ${ }^{14,15}$. Isto, muitas vezes, deve-se à falta de adequação das palavras e expressões utilizadas no termo, que em alguns casos reproduzem o jargão científico. Considere-se, ademais, a pouca escolaridade da maioria dos participantes, fator que dificulta a leitura fluente, a compreensão de períodos longos e de palavras que fogem à linguagem coloquial ${ }^{16}$.

Um destes estudos mostrou que a utilização de recursos audiovisuais pode facilitar a comunicação com os pacientes: observou que aqueles aos quais foi oferecida esta mídia apresentaram maior entendimento sobre o estudo quando comparados com os pacientes do grupo que não fez uso do recurso ${ }^{6}$. Sobre formas alternativas para facilitar a compreensão do termo de consentimento pelos participantes, Abd-Elsayed et al descreveram recentemente que termos de consentimentos em formato realçado ou destacado - por exemplo, utilizando textura diferente de papel ou, até mesmo, formato chamativo - não facilitaram a compreensão dos pacientes que participaram de estudos clínicos ${ }^{17}$.

A obtenção de TCLE em estudos clínicos conduzidos na área da oncologia pediátrica apresenta aspectos peculiares à população aos quais se dirigem: crianças e adolescentes. É importante ponderar que nesta faixa etária os pacientes são emocionalmente mais inseguros e vulneráveis às adversidades, o que é intensificado pelo impacto do diagnóstico de câncer. Haja vista a ausência de capacidade legal da criança e do adolescente para fornecer o seu consentimento, 0 documento é assinado pelos pais ou representantes legais. Entretanto, além do consentimento destes fazse preciso obter o assentimento livre e esclarecido da própria criança, considerando sua capacidade de compreensão. No Brasil, a participação de crianças e adolescentes em estudos clínicos é também regulamentada pela Resolução CNS 196/96 ${ }^{10}$.

\section{Protocolo brasileiro para tratamento de sarco- ma de Ewing}

O protocolo brasileiro para tratamento dos pacientes portadores de tumores da família do sarcoma de Ewing foi elaborado em 2004 a partir do consenso entre pesquisadores de instituições dedicadas ao atendimento de crianças com câncer. Este grupo entendeu que, considerando que nossos pacientes portadores de sarcoma de Ewing são diagnosticados, em geral, quando a doença encontra-se em estágio avançado, quando comparados aos pacientes dos países da América do Norte ou da Europa, seria necessário elaborar e aplicar um programa de tratamento adaptado à realidade brasileira e, posteriormente, à uruguaia. Basicamente, o protocolo recomendava o tratamento de acordo com o grupo de risco, ou seja, os pacientes com doença mais avançada, grupo denominado como de alto risco, recebiam tratamento quimioterápico adicional quando comparados aos pacientes pertencentes ao grupo de baixo risco, que recebiam o tratamento convencional.

O objetivo deste estudo foi avaliar o cumprimento dos aspectos éticos e normativos de um protocolo de experimentação clínica multicêntrico em oncologia pediátrica.

\section{Materiais e método}

Trata-se de estudo transversal retrospectivo. 0 universo de pesquisa foi composto por 180 pacientes 
portadores de sarcoma de Ewing procedentes de 15 centros de pesquisa no Brasil e um no Uruguai - totalizando 16 centros participantes. A coordenação do estudo é constituída por pesquisadores brasileiros e a equipe de monitores integra a unidade de monitoração de estudos clínicos (Umec) do centro de pesquisa de um dos membros da coordenação. Os dados foram coletados a partir das informações registradas nas fichas clínicas e prontuários médicos dos pacientes, atendidos durante o período de 2004 a 2010.

O projeto protocolo brasileiro para o tratamento de pacientes com tumores da família do sarcoma de Ewing foi elaborado por um grupo que incluiu oncologistas pediátricos, cirurgiões ortopédicos, radioterapeutas e patologistas com o objetivo de avaliar se o programa terapêutico proposto seria promissor em relação à resposta tumoral e ao perfil de segurança. O desenho do estudo consistiu na classificação de cada paciente em um dos dois grupos de risco - baixo risco ou alto risco -, considerando os principais aspectos clínicos que apresentam influência prognóstica.

Os critérios utilizados para caracterizar os pacientes como de alto risco consideraram a presença de pelo menos um dos seguintes itens: nível sérico de LDH 2,5 vezes acima do limite superior; tumor primário de localização pélvica; presença de metástases; ou tumor irressecável. Os demais pacientes foram alocados no grupo de baixo risco. Este estudo clínico recomendou três avaliações clínicas em momentos diferentes. A primeira, antes de iniciar o tratamento; a segunda, após a fase chamada "indução" e a terceira, após o término do tratamento. Com estas informações seria possível comparar os exames de forma evolutiva. A resposta tumoral obtida neste estudo foi analisada de acordo com os critérios recomendados pelo Response Evaluation Criteria In Solid Tumours (Recist). A avaliação das toxicidades considerou os critérios do Common Toxicity Criteria versão 3.0, usualmente utilizado para avaliar eventos adversos em pacientes tratados com quimioterapia.

Uma vez definido o esquema de tratamento, os membros coordenadores do estudo redigiram e enviaram o protocolo para aprovação do CEP do Hospital de Clínicas de Porto Alegre (HCPA). Após sua aprovação foram selecionados os centros de oncologia pediátrica que seriam convidados a participar do protocolo. A escolha considerou os centros cujo corpo clínico apresentava um oncologista pe- diátrico e um ortopedista com experiência em tumores ósseos, além da disponibilidade de exames de imagem como tomografia computadorizada e/ ou ressonância magnética e das drogas quimioterápicas indicadas pelo estudo. Também foi considerada a presença de toda equipe de suporte que envolve o atendimento de crianças e adolescentes com câncer.

Após a identificação dos centros com critérios para participar do estudo, a coordenação encaminhou convite formal, por telefone e/ou e-mail, para os respectivos investigadores. Posteriormente, um monitor de pesquisa fez contato com cada pesquisador com potencial interesse e reforçou as informações relativas à proposta do protocolo, incluindo seus objetivos, critérios de inclusão e exclusão, desenho do estudo, tratamento e parâmetros de avaliação de resposta. Este profissional também explicou como seriam os procedimentos regulatórios que consistem na submissão do projeto ao CEP, nos procedimentos éticos que incluem principalmente a aplicação do TCLE, nos registros dos dados nas fichas clínicas e nos procedimentos das visitas de monitoria ao longo do estudo.

Os centros que concordaram em participar do protocolo receberam a visita da equipe de monitoria para esclarecer as dúvidas do pesquisador e da equipe, antes da inclusão de pacientes. Neste encontro foi também avaliada a infraestrutura de cada centro de pesquisa, a capacitação dos profissionais envolvidos, a capacidade de recrutamento de pacientes e as dúvidas em relação ao preenchimento das fichas clínicas, além da obtenção de informações sobre o CEP local. Todos os centros colaboradores participantes do estudo receberam por meio eletrônico a versão escrita do protocolo, do TCLE e das fichas clínicas para submetê-las à apreciação dos CEP locais.

A coordenação estabeleceu que somente após a aprovação pelo CEP local a instituição poderia incluir pacientes no estudo. Uma vez iniciada a inclusão de pacientes, os monitores de pesquisa realizaram visitas às instituições analisando as fichas clínicas, os documentos do arquivo do investigador, os documentos submetidos ao CEP local e os documentos-fonte, como os prontuários médicos. Todas as informações e pendências a serem resolvidas na próxima visita eram registradas nas cópias das fichas clínicas de cada paciente - mantidas no arquivo do centro coordenador do estudo. 
Com o estudo clínico em andamento, um centro uruguaio foi convidado a participar do estudo - convite que considerou sua proximidade geográfica, a potencial inclusão de significativo número de pacientes e as afinidades com o centro coordenador, observadas em experiências prévias com outros protocolos. O fato de a língua oficial daquele país ser o espanhol não foi considerado empecilho à participação neste protocolo.

\section{Resultados}

A maioria dos 180 pacientes era do sexo masculino, com faixa etária entre 0 e 28 anos, cor da pele branca, com doença localizada e pertencente ao grupo de alto risco, conforme os critérios do protocolo. As características gerais dos pacientes estão descritas na Tabela 1.

Tabela 1. Características dos pacientes e da doença ( $N=180)$

\begin{tabular}{|c|c|}
\hline Características & $\mathbf{N}(\%)$ \\
\hline Idade no diagnóstico em anos & \\
\hline Mediana & 12 \\
\hline Variação & $0,2-28,9$ \\
\hline$<14$ anos & $108(60)$ \\
\hline$\geq 14$ anos & $72(40)$ \\
\hline Sexo & \\
\hline Masculino & $109(60,5)$ \\
\hline Feminino & $71(39,5)$ \\
\hline Cor da pele & \\
\hline Branca & $148(82,2)$ \\
\hline Outra & $32(17,8)$ \\
\hline Análise histológica & \\
\hline Sarcoma de Ewing & $86(47,7)$ \\
\hline Tumor neuroectodérmico primitivo & $69(38,3)$ \\
\hline Tumor de Askin & $18(10)$ \\
\hline Outra & $7(4)$ \\
\hline Grupo de risco & \\
\hline Alto & $127(70,5)$ \\
\hline Baixo & $53(29,5)$ \\
\hline Estágio da doença & \\
\hline Localizada & $110(61,2)$ \\
\hline Metastática & $70(38,8)$ \\
\hline
\end{tabular}

Submissão do protocolo de pesquisa e TCLE ao CEP

Dezesseis instituições incluíram pacientes neste estudo clínico. Dez (62,5\%) centros submeteram o protocolo de pesquisa e respectivo TCLE ao CEP local, que os aprovou. Os pesquisadores responsáveis por estes centros enviaram para a unidade de pesquisa do centro coordenador, via e-mail ou fax, a carta de aprovação emitida pelo CEP local. Apesar da recomendação do centro coordenador, cinco $(31,2 \%)$ centros de pesquisa brasileiros e o centro uruguaio $(6,3 \%)$ não submeteram o projeto a um CEP local ou regional.

\section{Assinatura do TCLE}

Na revisão dos relatórios realizados pelos monitores de pesquisa foi observado que 161 dos 180 $(89,4 \%)$ pacientes e/ou seus representantes legais consentiram e assinaram o TCLE aplicado pelo pesquisador responsável ou pessoas por ele designadas. Destes 161 casos, 123 (76,4\%) assinaram o TCLE específico do protocolo previamente aprovado pelo CEP e $38(23,6 \%)$ consentiram o tratamento mediante assinatura do TCLE próprio de cada instituição - destes 38 pacientes, $23(60,5 \%)$ estavam em atendimento na instituição uruguaia e 15 (39,5\%) em centros no Brasil. 
Em relação à data da assinatura do consentimento, $141(87,6 \%)$ pacientes e/ou seus responsáveis assinaram o TCLE após receberem as informações referentes ao estudo clínico proposto. Entretanto, a data da assinatura do TCLE de 20 pacientes $(12,4 \%)$ é posterior ao início do tratamento.
As visitas de monitoria não encontraram qualquer tipo de TCLE, quer específico do protocolo ou institucional, nos prontuários de 19 (10,5\%) pacientes. Estes pacientes estavam distribuídos em sete diferentes instituições. Os resultados acima descritos referentes ao TCLE estão resumidos nas Tabelas 2 e 3.

Tabela 2. Assinatura do termo de consentimento livre e esclarecido $(\mathrm{N}=180)$

\begin{tabular}{|l|c|}
\multicolumn{1}{c}{ Assinatura } & $\mathbf{N}(\%)$ \\
TCLE do protocolo & $123(76,4 \%)$ \\
TCLE institucional & $38(23,6 \%)$ \\
Uruguai & $23(60,5 \%)$ \\
Brasil & $15(39,5 \%)$ \\
Não assinaram o TCLE & $19(10,5 \%)$ \\
\hline
\end{tabular}

Tabela 3. Momento da assinatura do termo de consentimento livre e esclarecido ( $N=161)$

\begin{tabular}{|l|c|}
\hline \multicolumn{1}{|c|}{ Assinatura } & $\mathbf{N}(\%)$ \\
\hline Antes do início do tratamento & $141(87,6 \%)$ \\
Após o início do tratamento & $20(12,4 \%)$ \\
\hline
\end{tabular}

\section{Discussão}

Os índices de sobrevida dos pacientes portadores de câncer infantojuvenil têm aumentado significantemente nos últimos anos, resultado do conjunto de ações que inclui melhoria nos cuidados básicos, disponibilidade de exames laboratoriais e de imagem mais acurados e a utilização de tratamentos mais eficazes identificados pelos estudos clínicos multicêntricos. O câncer infantojuvenil é condição considerada rara, portanto, somente com a condução de estudos envolvendo diversas instituições é possível obter significativo número de pacientes para atender seus objetivos em período razoavelmente curto de observação.

Atualmente, tanto os estudos clínicos cooperativos quanto os coordenados e patrocinados pela indústria farmacêutica devem obedecer os mesmos padrões éticos, científicos e de qualidade. Os estudos que envolvem registros de novos medicamentos devem ser sujeitos à monitoração, auditoria e inspeções por terceiros. Nos estudos clínicos que envolvem somente questões assistenciais inerentes ao atendimento clínico de rotina dos pacientes o médico assistente deve obedecer aos preceitos da boa prática clínica, conduta que deve ser seguida independentemente se o paciente está ou não incluído em algum protocolo de estudo.

Existem muitos estudos cooperativos que agregam importantes conhecimentos sobre a se- gurança e eficácia de uma ou mais drogas aprovadas. Em alguns países, como os Estados Unidos, por exemplo, esses estudos apresentam monitoração contínua por profissionais do próprio centro coordenador, como evidenciado por diversos autores que manifestam preocupação com a qualidade dos dados ${ }^{18-22}$. Alguns estudos descritivos demonstraram a importância de treinamento específico para as equipes de pesquisa que conduzem estudos clínicos na área da oncologia ${ }^{19,20}$.

A visita de monitoramento influencia de forma eficaz a qualidade da condução destes estudos e tem como aliado o conhecimento e a experiência dos investigadores ${ }^{20}$. Knatterud et al criaram um guia para padronizar o controle de qualidade de estudos multicêntricos, incluindo recomendações de ações apropriadas para os problemas que mais frequentemente ocorrem durante um estudo clínico ${ }^{21}$.

É importante salientar que além da preocupação em relação à confiabilidade dos dados coletados em um estudo clínico, outro aspecto igualmente importante, que também necessita atenção especial, é a adequação do estudo às recomendações éticas, legais e científicas da legislação vigente. Estes aspectos têm sido foco de atenção dos estudos cooperativos na área da oncologia pediátrica - não somente nos desenvolvidos no Brasil, mas também nas instituições de todo o mundo.

Em relação aos aspectos éticos e legais discutidos neste artigo, toda e qualquer pesquisa clínica 
envolvendo seres humanos deve seguir as normas e diretrizes internacionais e nacionais. Após o planejamento e delineamento da pesquisa, o protocolo de pesquisa e o TCLE devem ser avaliados e aprovados por um CEP e, em algumas situações, é necessária a avaliação pela Conep.

No estudo clínico, o centro coordenador submeteu o protocolo de pesquisa e respectivo TCLE ao CEP local. Após a aprovação, foi enviada cópia do protocolo e do TCLE para todas as instituições interessadas em participar no estudo, para que a submetessem ao seu CEP. Esta documentação foi enviada via e-mail ou correio, trazendo anexa a carta de aprovação do CEP do HCPA. Os centros candidatos foram informados que se ocorresse alguma dúvida sobre o trâmite regulatório os monitores de pesquisa estariam à disposição para esclarecimentos. Adicionalmente, a coordenação do estudo clínico insistentemente recomendava às instituições candidatas que enviassem o protocolo de pesquisa e seu respectivo TCLE para aprovação nos CEP locais.

Cinco centros de pesquisa brasileiros não enviaram o projeto para aprovação nos CEP locais. A justificativa da não realização deste procedimento não tem registro nos relatórios de monitoria. Os motivos para que alguns dados ficassem incompletos e de difícil resgate pela equipe atualmente em atividade foram: a) em 2004, quando o protocolo foi instituído, muitos pesquisadores ainda não estavam familiarizados com a tramitação de pesquisa clínica; b) trata-se de um protocolo de tratamento que recomendava a utilização de esquema de quimioterapia muito semelhante ao tratamento assistencial; c) em algumas instituições colaboradoras houve mudanças no pessoal envolvido com o projeto; d) é também possível que estes centros habitualmente não apresentassem a rotina de enviar projetos para apreciação ao CEP local; e) os monitores não conheciam ou não estavam habituados a acompanhar tal requisito.

Em 2004, o centro de pesquisa do Uruguai não submeteu o projeto ao comitê de ética pois a legislação daquele país não exigia a aprovação de projetos desta natureza por um CEP. No entanto, em 2008, entrou em vigor o Decreto 379/08 do Poder Executivo do Ministério da Saúde Pública do Uruguai muito similar à Resolução CNS 196/96. Os itens 26 e 28 do Capítulo VI deste decreto especificam, respectivamente, que toda a investigação deverá ser submetida à apreciação de um Comitê de Ética em Investigação e; se houver a impossibilidade de constituir um Comitê de Ética em Investigação, a instituição deverá apreciar o seu projeto em outra instituição que tenha um Comitê de Ética em Inves- tigação, observando as indicações da Comissão Nacional de Ética em Investigação ${ }^{23}$. Portanto, como o planejamento e o trâmite regulatório do estudo clínico tiveram início em 2004 e esta determinação passou a vigorar posteriormente, em 2008, o centro de pesquisa do Uruguai aplicou o protocolo sem submetê-lo ao comitê de ética. Salienta-se, ainda, que no citado decreto não houve qualquer menção à submissão de projetos em andamento, iniciados antes de 2008. Curiosamente, a instituição uruguaia contribuiu de maneira satisfatória, tendo aplicado o TCLE institucional para todos os pacientes participantes e apresentado o menor número de pendências durante todo o período do estudo, além de ter incluído significativo número de pacientes.

O processo de obtenção do TCLE define que o paciente ou seu responsável sejam informados sobre os riscos, desconfortos ou benefícios que o estudo clínico pode lhe proporcionar, visando, assim, preservar o princípio de autonomia no que diz respeito a sua participação. No Brasil, a aplicação do TCLE na pesquisa clínica fundamenta-se na Resolução CNS 196/96, além das normas e diretrizes internacionais. Cada centro de pesquisa deve elaborar o seu próprio TCLE para as atividades de rotina. Porém, em estudos clínicos multicêntricos nacionais ou internacionais, deve-se adotar um modelo único para todas as instituições participantes, evitando que ocorram discrepâncias na elaboração do documento. Na pesquisa clínica somente o TCLE redigido especificamente para o projeto (e posteriormente aprovado pelo (EP) tem valor regulatório.

Com base na Resolução CNS 196/96, o TCLE do projeto foi elaborado pelo pesquisador responsável pelo estudo clínico, cumprindo todas as exigências da legislação. Neste estudo clínico, todos os centros de pesquisa foram orientados a utilizar o mesmo TCLE do projeto. Trinta e oito pacientes participantes firmaram o consentimento por meio do TCLE institucional, ressaltando-se que o mesmo só é válido para atividades assistenciais de rotina. Entretanto, em um estudo clínico, se o pesquisador optar pelo TCLE institucional deve incluir as informações específicas referentes à pesquisa em questão, versão que deve ser submetida à aprovação pelo CEP. A utilização do TCLE institucional para aplicação de quimioterapia foi considerada um desvio de protocolo e esta conduta irregular foi registrada nos relatórios de monitoria dos centros.

O item c da diretriz IV.2 da Resolução CNS 196/96 diz que o TCLE deve ser assinado ou identificado por impressão dactiloscópica, por todos e cada um dos sujeitos da pesquisa ou por seus repre- 
sentantes legais. Vale ressaltar que a assinatura é importante, porém o fundamental é o processo de obtenção do consentimento que, necessariamente, deve ser precedido pelo fornecimento de informação precisa. É obrigatório que o pesquisador responsável ou sua equipe certifiquem-se que todos os participantes da pesquisa não só tenham assinado o TCLE específico da pesquisa, mas, de fato, entendido seu conteúdo totalmente. No estudo clínico em questão, verificou-se que 19 pacientes ou seus representantes legais, atendidos em sete instituições diferentes, não assinaram o TCLE. Esta atitude é uma violação ao protocolo e a situação destes pacientes deveria ser avaliada individualmente para que se entenda a razão exata da ausência do TCLE.

Entretanto, como anteriormente dito, o motivo pelo qual estes pacientes não assinaram o consentimento não ficou registrado nos prontuários médicos ou nos relatórios de monitoria. É provável que esta falha possa ser creditada ao fato da apresentação do TCLE não ser rotina nestes centros. Como estes pacientes receberam o tratamento conforme preconizado no protocolo, a coordenação do estudo, neste caso, considerou esta amostra e não excluiu os da análise dos resultados porque os dados coletados destes pacientes poderiam vir a contribuir para achados clínicos futuros. Nota-se, entretanto, que ainda é necessário aprofundar o conhecimento e aprimorar a prática dos pesquisadores acerca dos aspectos relacionados ao processo de obtenção do consentimento informado em estudos que envolvam seres humanos ${ }^{23}$.

A diretriz IV.4.a da Resolução CNS 196/96 preconiza acerca da restrição à liberdade e do esclarecimento necessários para o adequado consentimento do participante de pesquisa em situação de substancial diminuição em suas capacidades de consentimento. A resolução indica que nestes casos deverão ser cumpridas as exigências do consentimento livre e esclarecido, por meio dos representantes legais dos referidos participantes, mantendo o direito de informação do convidado, no limite de sua capacidade ${ }^{10}$. Quando as pesquisas são realizadas com crianças e adolescentes, estes devem participar ativamente do processo de obtenção do consentimento. Neste caso, dois tipos de consentimento serão elaborados para a pesquisa clínica: um termo de assentimento para o menor de idade (sendo convidado a participar da pesquisa) e um termo de consentimento para o responsável legal (sendo informado que o menor está sendo convidado para participar da pesquisa e solicitando a sua autorização).
Assim, é atualmente mandatório que todo paciente envolvido em estudos clínicos na área da oncologia pediátrica também participe do consentimento mediante assinatura do termo de assentimento - obviamente, desde que saiba escrever o próprio nome. No protocolo do estudo clínico não constava o termo de assentimento para as crianças e adolescentes.

Como o TCLE ficou arquivado no centro de pesquisa correspondente, não ficou registrado no centro coordenador se o TCLE foi assinado pelos pais ou pelo paciente. Conforme citado, o adequado seria incluir um termo de assentimento para o menor, além do TCLE para seu representante legal. Foi constatado, ainda, que 20 pacientes assinaram o TCLE após o início da administração das drogas quimioterápicas. Entretanto, todos receberam do pesquisador responsável, antes de iniciar o tratamento, conforme consta nas evoluções dos prontuários, uma explicação verbal sobre a doença e o tratamento proposto pelo protocolo brasileiro.

Enfim, os desvios e as violações descritas neste artigo foram entregues à coordenação do estudo e à equipe de monitores. Podemos concluir que as não conformidades encontradas possam estar relacionadas, principalmente, com a mudança de membros da equipe dos centros de pesquisa, com a falta de conhecimento dos trâmites regulatórios e normativos e com a atenção ou comunicação insuficiente entre o monitor de pesquisa e a equipe assistencial. Notou-se que a maioria dos relatórios de monitoria foi redigida de maneira objetiva, mas somente algumas pendências foram descritas de forma mais detalhada. Este é um aspecto relevante e que pode interferir na análise dos dados, haja vista que a riqueza das informações contribui para uma interpretação mais adequada de uma pesquisa clínica, além de permitir identificar alterações e agir de maneira imediata com ações corretivas. Por este motivo, especialmente em projetos multicêntricos, uma monitoria mais rigorosa, em termos de orientação e fiscalização do trâmite regulatório como no processo de obtenção do TCLE e do termo de assentimento, poderia prevenir situações como as encontradas.

\section{Considerações finais}

Nos últimos anos, os estudos clínicos se mostraram de extrema importância para a identificação de novas drogas e esquemas terapêuticos na área da oncologia pediátrica. Com o crescimento do número de estudos clínicos nesta área, é fundamental 
que os pesquisadores estejam cada vez mais familiarizados com os aspectos éticos, legais e regulatórios da pesquisa clínica para que os estudos assegurem a dignidade e o respeito aos participantes.

A coordenação do estudo compreende que houve alguns problemas relacionados com estes aspectos, o que já era previsto, dado que a pesquisa envolveu significativo número de instituições de diversos locais do país e do exterior, cada uma com suas peculiaridades nas rotinas de atendimento. Outra questão relevante é que, à época, muitas instituições não estavam ainda familiarizadas com aspectos regulatórios de uma pesquisa envolvendo seres humanos.

Apesar de ser uma pesquisa terapêutica destinada a avaliar se o programa proposto seria promissor em relação à resposta tumoral e ao perfil de segurança, é indispensável reiterar que o tratamento preconizado no protocolo de pesquisa é o mesmo atendimento padrão fornecido em todas as instituições que participaram do estudo. Assim, se não houvesse este estudo clínico os pacientes portadores de sarcoma de Ewing atendidos nestas instituições receberiam o mesmo esquema de tratamento proposto, porém não seriam beneficiados pelo controle de qualidade de dados. Portanto, consideramos que, mesmo com as irregularidades éticas e regulatórias identificadas ao longo deste artigo, a condução de um estudo clínico nesta área deve considerar os potenciais benefícios aos pacientes, especialmente nos casos cuja terapêutica já tenha sido comprovada em termos de segurança, eficácia e efetividade, como ocorreu neste estudo.

Nessas circunstâncias, é importante considerar como parâmetro ético de avaliação do estudo a relação risco/benefício, que no presente caso se mostrou favorável, considerando-se que por ser terapêutica padrão o estudo não implica em risco adicional e traz os benefícios do controle de qualidade de dados e do acompanhamento metódico dos efeitos das drogas quimioterápicas pela equipe de monitoria.

Sabemos que é obrigatório ter a aprovação do projeto de pesquisa pelo CEP/Conep e do processo de obtenção do TCLE. Estas exigências têm sido discutidas em diversos congressos médicos e também em encontros sobre pesquisa clínica no Brasil e no mundo. O cumprimento das normas éticas, regulatórias e técnicas são fundamentais para a boa condução de uma experimentação clínica, pois concorrem para a difusão do conhecimento e da experimentação científica aos profissionais envolvidos e à sociedade. Além disso, este cumprimento aumenta o grau de segurança e de proteção dos pacientes participantes, o grau de qualificação das instituições participantes e estimula a aderência de pacientes ao protocolo de pesquisa proposto.

Por fim, os autores reconhecem e agradecem o esforço e participação de cada centro de pesquisa.

\section{Referências}

1. Smith MA, Seibel NL, Altekruse SF, Ries LA, Melbert DL, O'Leary M et al. Outcomes for children and adolescents with cancer: challenges for the twenty-first century. J Clin Oncol. 2010;28(15):262534.

2. Kaatsch P. Epidemiology of childhood cancer. Cancer Treat Rev. 2010;36(4):277-85.

3. Murphy SB. The national impact of clinical cooperative group trials for pediatric cancer. Med Ped Oncol. 1995;24:279-80.

4. Wagner HP, Dingeldein-Bettler I, Berchthold W, Luthy AR, Hirt A, Pluss HJ et al. Childhood NHL in Switzerland: incidence and survival of 120 study and 42 non-study patients. Med Ped Oncol. 1995;24(5):281-6.

5. Organização Pan-Americana da Saúde. Boas práticas clínicas: documento das Américas. IV Conferência Pan-Americana para Harmonização da Regulamentação Farmacêutica. República Dominicana 2-4 de março de 2005. Disponível: http://www.anvisa.gov.br/medicamentos/ pesquisa/boaspraticas_americas.pdf (acesso nov. 2012)

6. Goldim JR. A avaliação do projeto de pesquisa: aspectos científicos, legais, regulatórios e éticos. Rev HCPA \& Fac Med Univ Fed Rio Gd do Sul. 2006;26(1):83-6.

7. Goldim JR, Pithan CF, Oliveira JG, Raymundo MM. O processo de consentimento informado livre e esclarecido em pesquisa: uma nova abordagem. Rev Assoc Med Bras. 2003;49(4):372-4.

8. Associação Médica Mundial. Declaração de Helsinki I. [internet]. Adotado pela $18^{a}$ Assembleia Geral da Associação Médica Mundial, Helsinque, Finlândia, junho 1964 e emendas. [acesso 14 set. 2011]. Disponível: http://www.bioetica.ufrgs.br/helsin1.htm

9. Conselho Nacional de Saúde (Brasil). Resolução $n^{\circ} 1$, de 13 de junho de 1988. Aprova normas de pesquisa em saúde. [internet]. [acesso 14 set. 2011]. Disponível: http://conselho.saude.gov.br/ resolucoes/ 
10. Brasil. Ministério da Saúde. Conselho Nacional de Saúde. Resolução n 196/96, de 10 de outubro de 1996. Diretrizes e normas regulamentadoras de pesquisa envolvendo seres humanos. Disponível: http://conselho.saude.gov.br/web_comissoes/conep/aquivos/resolucoes/23_out_ versao_final_196_ENCEP2012.pdf (acesso 26 out. 2012).

11. Francisconi CF, Kipper DJ, Clotet J, Oselka G, Goldim JR. Comitês de ética em pesquisa: levantamento de 26 hospitais brasileiros. Bioética. 1995;3(1):61-7.

12. International Conference on Harmonisation of Technical Requirements for Registration of Pharmaceuticals for Human Use. ICH harmonized tripartite guideline for good clinical practice (GCP). [internet]. 1996 [acesso mar. 2013]. Disponível: http://www.ich.org/fileadmin/Public Web_Site/ICH_Products/Guidelines/Efficacy/E6_R1/Step4/E6_R1_Guideline.pdf

13. Hereu P, Pérez E, Fuentes I, Vidal X, Suñé $P$, Arnau JM. Consent in clinical trials: what do patients know? Contemp Clin Trials. 2010;31(5):443-6.

14. Pandiya A. Readability and comprehensibility of informed consent forms for clinical trials. Patient Educ Couns. 2012;87(2):243-9.

15. Crepeau AE, McKinney BI, Fox-Ryvicker M, Castelli J, Penna J, Wang ED. Prospective evaluation of patient comprehension of informed consent. J Bone Joint Surg Am. 2011;93(19):e114(1-7).

16. Abd-Elsayed AA, Sessler DI, Mendoza-Cuartas M, Dalton JE, Said T, Meinert J et all. A randomized controlled study to assess patients' understanding of and consenting for clinical trials using two different consent form presentations. Minerva Anestesiol. 2012;78(5):564-73.

17. Pollock BH. Quality assurance for interventions in clinical trials: multicenter data monitoring, data management, and analysis. Cancer. 1994;74(9 Suppl):2.647-52.

18. Favalli G, Vermorken JB, Vantongelen K, Renard J, Van Oosterom AT, Pecorelli S. Quality control in multicentric clinical trials: an experience of the Eortc Gynecological Cancer Cooperative Group. Eur J Cancer. 2000;36(9):1.125-33

19. van der Putten E, van der Velden JW, Siers A, Hamersma EA. A pilot study on the quality of data management in a cancer clinical trial. Control Clin Trials. 1987;8(2):96-100.

20. Vantongelen K, Rotmensz N, van der Schueren E. Quality control of validity of data collected in clinical trials. Eortc Study Group on Data Management (SGDM). Eur J Cancer Clin Oncol. 1989;25(8):1.241-7.

21. Knatterud GL, Rockhold FW, George SL, Barton FB, Davis CE, Fairweather WR et al. Guidelines for quality assurance in multicenter trials: a position paper. Control Clin Trials. 1998;19(5):477-93.

22. Uruguay. Ministerio Salud Pública. Decreto $n^{\circ} 379 / 08$, de 4 de agosto de 2008. Investigación com seres humanos. [internet]. [acesso 3 nov. 2011]. Disponível: http://www.elderechodigital.com. uy/smu/legisla/D0800379.html

23. Hardy E, Bento SF, Osis MJD. Consentimento livre e esclarecido: experiência de pesquisadores brasileiros na área da regulação da fecundidade. Cad. Saúde Pública. 2004;20(1):216-23.

\section{Participação dos autores}

Maryelle Gamboa foi responsável pela concepção do artigo, revisão bibliográfica e redação. Lauro Gregianin, pela concepção do artigo e revisão.

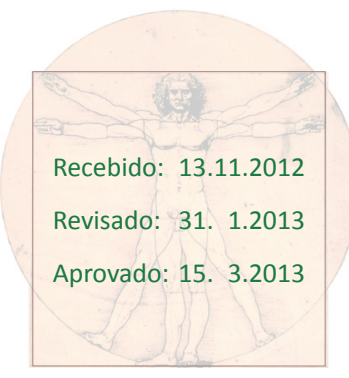

